\title{
Risk of Pyogenic Liver Abscess in Proton Pump Inhibitor Users: New Inventory of Proton Pump Inhibitor Induced Complications?
}

\author{
Ki Bae Bang \\ Department of Internal Medicine, Dankook University College of Medicine, Cheonan, Chungcheongnam-do, Korea
}

\author{
Article: Proton pump inhibitor use increases pyogenic liver abscess risk: a nationwide cohort study \\ Oh JH, Kang D, Kang W, Guallar E, Cho J, Min YW \\ (J Neurogastroenterol Motil 2021;27:555-564)
}

Proton pump inhibitors (PPIs) are widely used to manage acid-related gastrointestinal diseases, and the use of PPIs is increasing worldwide. Since the introduction of omeprazole in the 1980s, PPIs have gained popularity due to their proven efficacy and safety profile. ${ }^{1}$ In the United Kingdom, increasing prevalence of PPI use was reported in general practice from $0.2 \%$ to $15.0 \%$ between 1990 and $2014 .{ }^{2}$ In France, approximately one-third of the estimated adult population used PPIs in $2015 .^{3}$ In the United States, $8.0 \%$ to $10.0 \%$ of ambulatory adults have been prescribed PPIs in the past 30 days. ${ }^{4}$ PPIs suppress acid secretion by irreversibly inhibiting gastric $\mathrm{H}^{+} / \mathrm{K}^{+}$-adenosine triphosphatase. They are effective for the management of gastroesophageal reflux disease, peptic ulcer disease, and are used as first-line therapy for functional dyspepsia. ${ }^{1,5}$ Even higher doses of PPIs are used for the control of extraesophageal symptoms and Helicobacter pylori eradication therapy. ${ }^{6,7}$ Moreover, long-term management in patients with Barrett's esophagus is recommended to reduce the occurrence of dysplasia. In addition, concomitant administration of PPIs for the prevention of peptic ulcer disease in chronic users of NSAIDs and maintenance treatment in patients with gastroesophageal reflux disease is a common clinical practice. $^{4}$

With the increasing use of PPIs, concerns regarding the adverse effects of PPIs have been raised in recent epidemiologic studies. Complications associated with PPI use have been reported in various organs, including fundic gland polyps, microscopic colitis, acute interstitial nephritis, chronic kidney disease, myocardial infarction, fracture, community-acquired pneumonia, bacterial enteric infection with Clostridium difficile, hepatic encephalopathy, and spontaneous bacterial peritonitis in patients with cirrhosis, dementia, and gastrointestinal malignancies. ${ }^{8}$

Most evidence on complications related to PPI use is not based on well-designed studies. Confounding factors and causal relationships remain unresolved. ${ }^{9}$ Also, the reported risk of an adverse event is relatively low. ${ }^{10}$ However, it has been reported constantly through epidemiologic studies. Of these, increasing susceptibility to infection induced by PPI use has relatively strong clinical relevance. ${ }^{11}$ Several plausible pathophysiologic mechanisms of PPI-induced complications have been postulated. Most of the convincing adverse effects of PPIs are related to reduced gastric acid secretion. PPI-induced hypochondria can induce intestinal dysbiosis, enteric infection, and

Received: September 6, 2021 Revised: September 7, 2021 Accepted: September 17, 2021

(.) This is an Open Access article distributed under the terms of the Creative Commons Attribution Non-Commercial License (http://creativecommons. org/licenses/by-nc/4.0) which permits unrestricted non-commercial use, distribution, and reproduction in any medium, provided the original work is properly cited.

*Correspondence: Ki Bae Bang, MD, PhD

Department of Internal Medicine, Dankook University College of Medicine, 201, Manghyang-ro, Dongnam-gu, Cheonan,

Chungcheongnam-do 31116, Korea

Tel: +82-41-550-7630, Fax: +82-556-3256, E-mail: kibaebang@gmail.com 
defective absorption of micronutrients such as iron, calcium, and vitamin B12. ${ }^{10}$ Anti-neutrophilic effect of PPIs may contribute to increase the risk of infection. ${ }^{12}$

Regarding the risk of pyogenic liver abscess (PLA) in PPI users, little is known. Two population-based case-control studies were conducted on this issue in Taiwan, and an increased risk of PLA was reported. ${ }^{13,14}$ Because PLA is a prevalent disease, especially in Asia, it could be a matter in this population. ${ }^{15}$ In this issue of the Journal of Neurogastroenterology and Motility, Oh et al ${ }^{16}$ reported a retrospective cohort study evaluating the effects of PPIs on PLA with a follow-up period of 10 years. In this large nationwide population-based study ( $\mathrm{n}=58595)$, PPI use was associated with an increased risk of developing PLA. Despite the retrospective nature of the study design, this study controlled for confounding factors in detail and has clinical relevance and plausible pathophysiological background. Moreover, a strong association was observed between PPI use and the occurrence of PLA. The adjusted hazard ratio for PLA with PPI use was reported to be 3.88 (95\% CI, 2.54-6.92). Although it is limited to directly compare the risk of PLA with a previous case-control study using OR, the strength of association could be estimated. In this respect, the risk of PLA in the current study is comparable to a previous epidemiologic study conducted in Taiwan (adjusted OR, 4.7; 95\% CI, 2.9-7.8 for current PPI users). ${ }^{13}$ However, it is different from previous studies for several reasons. First, this was a longitudinal study with 6 months washout period. The incidence according to PPI use could be evaluated, strengthening the causal relationship compared to a case-control study. Second, efforts were made to control for confounding factors using propensity score matching, multivariate analysis, and stratified analysis. In addition, not only disease-related comorbidities but also various lifestyle parameters, including income, residential area, body mass index, smoking status, and alcohol intake, were adjusted, and stratified. Moreover, other potential biases such as immunosuppressants, antibiotics, and systemic corticosteroids were considered in the analysis. Third, to control the reverse causation bias, PLA cases occurring in the first 7 days after PPI treatment were excluded to avoid cases in which PPIs were used during the symptom period before the diagnosis of PLA. ${ }^{17}$ Fourth, the chance of exposure misclassification bias is low because PPIs are available only by prescription in Korea.

The limitation of this study is its retrospective design. Despite adopting strict inclusion and control of confounding factors, selection bias is unavoidable in this type of study design. In addition, analysis of the dose-response relationship was not available in this study. Interestingly, there was no duration response relationship between PPI exposure time and the risk of PLA in this study. This phenomenon has also been observed in other studies. In the metaanalysis, which included 26 studies, a higher risk of community acquired pneumonia was observed in short-term PPI users (OR, 2.10; 95\% CI, 1.39-3.16). ${ }^{18}$ In a recent nationwide cohort study conducted in Korea, current use of PPI ( $<1$ month) was associated with an increased risk of worse clinical outcome of COVID-19 (adjusted OR, 1.90; 95\% CI, 1.46-2.77). ${ }^{19}$ This result may have been caused by protopathic bias. However, in the current study, reverse causation bias was controlled by excluding the PLA cases occurring in the first 7 days, and sensitivity analysis excluding the initial 14 days revealed an increased risk of PLA (hazard ratio, 2.59; 95\% CI, 1.15-5.84). In this regard, even short-term use of PPIs may be related to the development of PLA and further studies are required to elucidate this issue.

\section{Financial support: None.}

\section{Conflicts of interest: None.}

\section{References}

1. Strand DS, Kim D, Peura DA. 25 years of proton pump inhibitors: a comprehensive review. Gut Liver 2017;11:27-37.

2. Othman F, Card TR, Crooks CJ. Proton pump inhibitor prescribing patterns in the UK: a primary care database study. Pharmacoepidemiol Drug Saf 2016;25:1079-1087.

3. Lassalle M, Le Tri T, Bardou M, et al. Use of proton pump inhibitors in adults in France: a nationwide drug utilization study. Eur J Clin Pharmacol 2020;76:449-457.

4. Targownik L. Discontinuing long-term PPI therapy: why, with whom, and how? Am J Gastroenterol 2018;113:519-528.

5. Oh JH, Kwon JG, Jung HK, et al. Clinical practice guidelines for functional dyspepsia in Korea. J Neurogastroenterol Motil 2020;26:29-50.

6. Park W, Hicks DM, Khandwala F, et al. Laryngopharyngeal reflux: prospective cohort study evaluating optimal dose of proton-pump inhibitor therapy and pretherapy predictors of response. Laryngoscope 2005;115:1230-1238

7. Jung HK, Kang SJ, Lee YC, et al. Evidence-based guidelines for the treatment of Helicobacter pylori infection in Korea 2020. Gut Liver 2021;15:168-195.

8. Malfertheiner P, Kandulski A, Venerito M. Proton-pump inhibitors: understanding the complications and risks. Nat Rev Gastroenterol Hepatol 2017;14:697-710.

9. Fossmark R, Martinsen TC, Waldum HL. Adverse effects of proton pump inhibitors-evidence and plausibility. Int J Mol Sci 2019;20:5203.

10. Vaezi MF, Yang YX, Howden CW. Complications of proton pump inhibitor therapy. Gastroenterology 2017;153:35-48.

11. Bavishi C, Dupont HL. Systematic review: the use of proton pump 
inhibitors and increased susceptibility to enteric infection. Aliment Pharmacol Ther 2011;34:1269-1281.

12. Laheij RJ, Sturkenboom MC, Hassing RJ, Dieleman J, Stricker BH, Jansen JB. Risk of community-acquired pneumonia and use of gastric acid-suppressive drugs. JAMA 2004;292:1955-1960.

13. Wang YP, Liu CJ, Chen TJ, Lin YT, Fung CP. Proton pump inhibitor use significantly increases the risk of cryptogenic liver abscess: a population-based study. Aliment Pharmacol Ther 2015;41:1175-1181.

14. Lin HF, Liao KF, Chang CM, Lin CL, Lai SW. Correlation between proton pump inhibitors and risk of pyogenic liver abscess. Eur J Clin Pharmacol 2017;73:1019-1025.

15. Jun JB. Klebsiella pneumoniae liver abscess. Infect Chemother 2018;50:210218.
16. Oh JH, Kang D, Kang W, Guallar E, Cho J, Min YW. Proton pump inhibitor use increases pyogenic liver abscess risk: a nationwide cohort study. J Neurogastroenterol Motil 2021;27:555-564.

17. Chen SC, Tsai SJ, Chen CH, et al. Predictors of mortality in patients with pyogenic liver abscess. Neth J Med 2008;66:196-203.

18. Lambert AA, Lam JO, Paik JJ, Ugarte-Gil C, Drummond MB, Crowell TA. Risk of community-acquired pneumonia with outpatient protonpump inhibitor therapy: a systematic review and meta-analysis. PLoS One 2015;10:e128004.

19. Lee SW, Ha EK, Yeniova AÖ, et al. Severe clinical outcomes of COVID-19 associated with proton pump inhibitors: a nationwide cohort study with propensity score matching. Gut 2021;70:76-84. 